\title{
Crohn's Disease and its Complications: Case Report
}

\author{
Antonio Carlos Barbosa Ramos Junior, Gabrielle Ribeiro Braga, Hayfa Georges Moreira El \\ Nabbout, Vitória Chagas Benevides* and Brian França dos Santos
}

Gastroenterology and Digestive System Surgery Academic League at Iguaçu University, Medicine School, Brazil

*Corresponding author: Vitória Chagas Benevides, Gastroenterology and Digestive System Surgery Academic League at Iguaçu University, - UNIG - Medicine School, Brazil

To Cite This Article: Hayfa Georges Moreira El Nabbout, Vitória Chagas Benevides, Brian França dos Santos, Antonio Carlos Barbosa Ramos Junior and Gabrielle Ribeiro Braga. Crohn's Disease and its Complications: Case Report. Am J Biomed Sci \& Res. 2021 - 11(5). AJBSR.MS.ID.001668. DOI: 10.34297/AJBSR.2021.11.001668.

Received: 阱 January 20, 2021; Published: 㘹 January 26, 2021

\section{Introduction}

Crohn's disease (CD) is an inflammatory chronic disease of the gastrointestinal tract of insidious appearance and is characterized by an inflammatory, transmural and skip lesion pattern (healthy areas interspersed between involved areas). It affects mainly the colon, the terminal ileum and the perianal region. It expresses clinically between periods of remission, with diarrhea, abdominal pain and unspecific symptoms [1-5]. The complications (obstruction, fistulae, abscesses, arthritis and cutaneous, joint, hepatic and ophthalmic lesions) are directly related to the gravity and chronicity of the disease [2,4]. CD has an undefined etymology but it's frequently related to family history $[2,3,5]$. It presents equally among men and women, having its bigger incidence in people between 20 and 30 years old. The treatment, surgical or clinical, reaches only an improvement in the patient's quality of life but it does not cure the disease.

\section{Case Report}

L.M.R., female, black, single, student, born in Rio de Janeiro, living in Nova Iguaçu states that she was hospitalized about five years ago due to a severe anemia. She had a blood transfusion done and was released shortly after. After three months, the patient states to have presented recurring urinary infections and, after having consulted with a nephrologist, was prescribed antibiotic therapy for the period of six months, having evolved into a severe case of diarrhea 30 days after the beginning of the treatment. As days went by, she also complained of anorexia, having seen medical assistance once more and got a colonoscopy done. The findings suggested Crohn's disease, but the diagnosis was not concluded. The patient was directed to a proctologist and, still symptomatic, received orientation to have several tests done, including magnetic resonance imaging (MRI), another colonoscopy and a complete blood count. She refused to have the tests taken and two months after evolved to a worsening of her general state with an important case of diarrhea (no blood, no purulent exudate and defecation up to 28 times a day) and weight loss. She was admitted again in January 2016, this time in an ICU, staying in nosocomial care for 15 days, when she was diagnosed with Crohn's disease. The patient states to have had an improvement in her general state when she begun corticosteroid and biological antibodies treatment but complains of the terrible quality of life due to the strong abdominal pains. She reports the use of many prescribed drugs and persistence of diarrhea. She also complains of recurring urinary tract infections. She reports being lactose intolerant since her childhood and having hereditary spherocytosis. She reports having sleep irregularities because she wakes up many times to defecate.

\section{Objective}

To review Crohn's disease, understanding its clinical aspects and manifestations as well as correctly directing the treatment.

\section{Methods}

This paper is an incorporated review, done in databases such as PudMed, SciELO (Scientific Electronic Library Online) and Google Scholar. We searched for the keywords "Doença de Crohn" and "Crohn's Disease". As well as separating files from the year 2015 up to the current year of 2020. Among the many literatures found, six original articles; three in Portuguese and three in English were selected for this extended abstract.

\section{Literature Review}

Crohn's disease is an inflammatory chronic bowel disease with the potential to affect from the mouth to the anus. It equally affects men and women [5]. It has its biggest incidence in the second and 
third decade of life, but it can occur at any age [3]. Its etiology is idiopathic but it is known not to be contagious. Nevertheless, literature reports a bigger risk in jewish and caucasian of northern European origin, besides having family history as a main risk factor [3]. "Its incidence in different members of the same family suggests a genetic predisposition, mainly among siblings. And this is also associated with the probability of having a more severe and precocious disease" [article 2]. The possibility of the disease being triggered by an infectious autoimmune disorder and environmental factors is discussed [1-4]. "Due to its unknown etiology there are theories involving bacterial infections, immunological factors, genetics, psychological disorders, nutritional diets, gastrointestinal allergies and environmental factors" [2].

Crohn's disease is characterized by an inflammatory transmural mechanism and is macroscopically recognized by a skip lesion pattern in the digestive tract, ulceration and/ or cobblestone-like lesions in more advanced states $[5,6]$. The more affected portions of the gastrointestinal tract are: the small intestine, the terminal ileum, the ascending colon and the anus [3,5]. It rarely affects the rectum. Besides from the local affection, in the long term, the patients evolve with systemic complications, corroborating the suspicion of involvement of an autoimmune mechanism. Other affected systems are "articular, ocular, dermatological, hepatobiliary, nephrological, hematological, vasculary, pancreatic, pulmonary and cardiac systems" $[2,4,5]$.

Patients oscillate between periods of crisis (that become more frequent and long lasting each time) and periods of remission [2]. "CD's progression in patients develops in a different way from patient to patient" [5]. The most common symptoms are abdominal cramps, diarrhea (bloody or not; regular with steatorrhea), vomiting, fever, asthenia, anemia and weight loss/ cachexia [2-5]. On imaging tests, CD may manifest in areas of fibrosis, low blood perfusion, also presenting slimming of walls and the presence of fistulae [3]. Besides the local manifestations from the chronic inflammation, there are also the systemic ones and their pathophysiology is also unknown.

As the main complications from $\mathrm{CD}$, we have aphthous ulcers, anemia, perianal fistulae, intestinal obstruction, palpable mass in right lower quadrant, joint pain, ankylosing spondylitis, erythema nodosum, kidney stone and/ or gallstone [2]. "In children, the complications appear in the form of retardation of physical development and growth" [4]. Among the less common complications, there are: colorectal cancer and gastrointestinal hemorrhage.

For the diagnosis are necessary, besides from the clinical manifestations, imaging exams, biochemical exams and a histopathological study of the biopsy material $[3,4,5,6]$. Among the imaging tests, the most common ones are the endoscopic (mainly the colonoscopy; endoscopy, recto sigmoidoscopy), magnetic resonance imaging, computed tomography scan (with contrast; capsule endoscopy mainly for the investigation of the disease' status on the level of the small intestine) and abdominal ultrasound (mainly in children) [4-6]. "The MRI was superior to the CT scan in the detection of stenosis and thickening of the ileal wall [6]. In a laboratory analysis, the histopathological searches the suggestive antibody, ASCA [4,5] while, for a rigorous clinical treatment, it is common to ask for a fecal calprotectin exam, to evaluate the intestinal inflammation quantitatively and qualitatively. Furthermore, "there can be found iron and vitamin B12 deficiency and an increase of C-reactive protein (CRP) $[4,6]$. The parasitology fecal test is also interesting so that a differential diagnosis can be done with intestinal parasitology [5].

The treatment is mainly clinical $[2,3,5]$, using aminosalicylate and folic acid, adding, according to the gravity, antibiotic therapy of broad spectrum, immunosuppressant drugs, TNF-alpha inhibitor and corticosteroid therapy [6]. "Laxatives, antidiarrheal, analgesic, iron supplement can also be used for symptom relief according to the patient's clinical status" [2]. Besides that, extra intestinal manifestations in consequence of the disease can be treated with a specialist. The dietary pattern must be healthy, preferably accompanied by a nutritionist to satisfy the nutritional needs, and to avoid and control the physical damages [2,4]. The surgical approaches become optional in cases where the patient does not respond to the clinical treatment of the complications or when the complication exposes the patient to an imminent death risk $[2,4,5]$. "It is estimated that around 70 to $90 \%$ of patients will need in some form to do the surgical treatment in a moment in their lives, from a simple anal abscess draining to the more complex resection of bowel parts [3]. It is of great importance that patients receive treatment with endoscopy, according to the clinical need and frequency of the crisis, collaborating to evaluate the answer to the treatment and possible evolution of the complications.

The embarrassment and debility of the patient frequently cause social disorders and low self-esteem. This fact, besides harming the patient's emotional state, also complicates the control of the disease, worsening the physical and mental state $[3,4]$. This way, it becomes urgent that the patients receive a multidisciplinary treatment including a psychologist, a gastroenterologist and other occupations, as complications of Crohn's disease appear.

\section{Discussion}

In the anamnesis it is observed the patient's clinical status that will be described, evaluating the signs of gravity. The clinical status of the disease varies, however its symptomatology is specific, being constituted [6] in its initial phase [4] mainly by diarrhea, abdominal pain and fever $[4,6]$. The patient can also present symptoms like [6]: fatigue, rectal bleeding, anorexia, anemia, acute abdomen and 
intestinal obstruction [6], reaching a nutritional deficiency and asthenia [4].

Extra intestinal manifestations are present in one third of the patients, being peripheral arthritis the most frequent one, followed by cutaneous, kidney, eye and hepatobiliary complications, uveitis, scleritis and deep venous thrombosis [4]. In kids, a retardation of physical development can also happen.

It is important to point out that the signs and symptoms are individualized, accompanying the patient's general state and the period in which the disease was diagnosed. According to the symptomatology of the disease, it can be classified as: light to moderate, moderate to severe and severe to fulminant, presenting signs and symptoms that are specific to each degree of the disease.

The patient that presents light to moderate form expresses as symptoms: diarrhea and abdominal pain and it can also be followed by weight loss. In the moderate to severe phase, the patient has fever, considerable weight loss, nausea, intermittent vomiting and can also manifest severe anemia. In the severe to fulminant phase, the patient presents manifestations such as: high fever, intestinal obstruction and/ or abscesses, vomiting and a considerable weight loss and severe anemia [3].

The diagnosis is done with the clinical suspicion, endoscopic evaluations and the histopathological [7]. The colonoscopy biopsy is the golden standard test to diagnose CD. The patient has as a result an intramural lesion of inflammatory pattern. The biopsy frequently shows ASCA antibodies and a noncaseating granuloma pattern. The endoscopic tests are also important in order to have a qualitative knowledge of the affection and its extension, as well as to observe the evolution of the disease. In more advanced cases, it is common to have an endoscopic finding known as "cobblestones", caused by fibrosis in the intestinal loops [6]. In histology, CD is presented by chronic, transmural and irregular inflammation with an increase of plasma cells and lymphocytes in the lamina propria, also showing noncaseating granulomas (cells that are present in chronic inflammation) and an irregular and discontinued crypta [7]. Another test that should be asked for a patient with a suspicion of Crohn's Disease, besides from the endoscopy and colonoscopy, are laboratory tests such as: complete blood count, testing for ASCA antibodies and stool tests. The complete blood count checks alterations such as anemia (caused by the digestive bleedings) and leukocytosis, suggesting inflammation. If the blood count indicates eosinophilia, a parasitic infection should be considered as a differential diagnosis. A positive result in a serological test for ASCA antibodies points out and contributes to a CD diagnosis. Clinical stool analysis (coproculture, parasites test and fecal calprotectin) are essential to the differential diagnosis. The fecal calprotectin test is very important to diagnose and monitor Crohn's disease. Calprotectin is responsible for transporting calcium and zinc in the small intestine and originates from neutrophils. For that reason, a high level of fecal calprotectin reveals qualitatively the level of inflammation of the affected intestinal loops. Another possible auxiliary exam to diagnose Crohn's disease is the detection with microRNA biomarker although this test is not pathognomonic [6].

Crohn's Disease does not have a cure and, with that, the treatment's goal is to minimize the physical and psychological damage caused by the disease. It is observed that with the reduction of the inflammation there is an improvement in quality of life, weight gain and symptom control $[3,4]$. New eating habits should be a part of the patient's lifestyle and for that, nutritional counselling is important. Pharmacological therapy is composed of antibiotics, anti-inflammatory, biologic probiotics and immunosuppressants [4]. Surgical intervention is only recommended in the more severe cases of the disease $[3,4]$. It is important to point out that each organism reacts in a particular manner to the drugs, surgeries and nutritional education and for that reason, the individualization of the treatment and a good patient-doctor communication is essential.

\section{Conclusion}

The importance of the diagnosis in the early stages of the disease is emphasized whether to discard other pathologies or to prevent the affection of the gastrointestinal tract as a whole, avoiding a prognostic damage to the patient and improving their quality of life. In time, to track malignant diseases, mainly intestine cancer that has inflammatory intestinal diseases as a risk factor.

\section{References}

1. Ribeiro, Iolanda Cristina Teixeira (2009) Doença de Crohn: etiologia, patogênese e suas Implicações na terapêutica. Tese de Doutorado. Universidade da Beira Interior.

2. Rodrigo Lopes Felipe, Maycon Sales David, Elaine Paula Mendonça Franqueiro, Márcia Alves Ferreira (2020) Doença de crohn: das causas ao trata-mento, uma revisão literária. e-RAC 8(1): 2018.

3. De Cássia Gonçalves Mariane, Gonçalves, Maycon Douglas Santana, Da Silva, Claudia Peres (2020) Doença de crohn: um estudo de caso. humanidades e tecnologia (FINOM) 1(23): 343-361.

4. Soares Julie Arsie, Da Silveira Schauren Juliana, Stroparo, Elenice (2018) Doença de crohn: revisão de literatura. Revista eletrônica biociências, biotecnologia e saúde 11(21): 78-83.

5. Gomes, Karla Nayara Cristina (2016) Doença de Crohn e seus principais aspectos.

6. Papacosta NG, Nunes GM, Pacheco RJ, Cardoso MV, Guedes VR (2017) Doença deCrohn: um artigo de revisão. Revista de Patologia do Tocantins 4(2):25-35. 